\title{
Liquid Matrix Deposition on Conductive Hydrophobic Surfaces for Tuning and Quantitation in UV-MALDI Mass Spectrometry
}

\author{
Magnus Palmblad and Rainer Cramer* \\ The BioCentre, The University of Reading, Reading, United Kingdom
}

With its highly fluctuating ion production matrix-assisted laser desorption/ionization (MALDI) poses many practical challenges for its application in mass spectrometry. Instrument tuning and quantitative ion abundance measurements using ion signal alone depend on a stable ion beam. Liquid MALDI matrices have been shown to be a promising alternative to the commonly used solid matrices. Their application in areas where a stable ion current is essential has been discussed but only limited data have been provided to demonstrate their practical use and advantages in the formation of stable MALDI ion beams. In this article we present experimental data showing high MALDI ion beam stability over more than two orders of magnitude at high analytical sensitivity (low femtomole amount prepared) for quantitative peptide abundance measurements and instrument tuning in a MALDI Q-TOF mass spectrometer. Samples were deposited on an inexpensive conductive hydrophobic surface and shrunk to droplets $<10 \mathrm{~nL}$ in size. By using a sample droplet $<10 \mathrm{~nL}$ it was possible to acquire data from a single irradiated spot for roughly 10,000 shots with little variation in ion signal intensity at a laser repetition rate of 5-20 Hz. (J Am Soc Mass Spectrom 2007, 18, 693-697) @ 2007 American Society for Mass Spectrometry

$\mathrm{M}$ atrix-assisted laser desorption/ionization (MALDI) mass spectrometry (MS) has been tremendously successful in the analysis of large (bio)molecules in fields such as proteomics [1, 2]. This success has been partially achieved through continuous improvements in the sample preparation and data acquisition techniques that have been introduced to overcome some of the disadvantages of the common solid-state MALDI matrices such as 2,5-dihydroxybenzoic acid (DHB) and $\alpha$-cyano-4-hydroxycinnamic acid (CHCA) [3-5]. The topography and morphology of solid-state MALDI samples can be highly inhomogeneous and significantly change upon laser irradiation, leading to large fluctuations in analyte ion signal intensities in MALDI MS [6-8]. These fluctuations impede the efficient tuning of MALDI mass spectrometers and, more important, severely limit the use of MALDI MS for quantitative measurements. Fluctuating ion beams make coupling with mass spectrometers difficult because they regularly require the adjustment of parameters that is often impossible to achieve in real time or retrospectively.

Recently, new liquid UV-MALDI matrices have been introduced, promising to be an effective alternative to

Published online January 16, 2007

Address reprint requests to Prof. Rainer Cramer, The University of Reading, The BioCentre, Whiteknights, PO Box 221, Reading RG6 6AS, UK. E-mail: r.k.cramer@rdg.ac.uk

* Department of Chemistry, The University of Reading, PO Box 224, RG6 6AD, United Kingdom. the crystalline MALDI samples by overcoming some of their inherent disadvantages. Primarily, these are ionic liquid matrices (ILMs) [9, 10] and, usually viscous, liquid matrices that are doped with UV light-absorbing chromophores [7, 11, 12]. However, only a handful of research groups have focused on the further development of liquid MALDI systems and so progress has been slow despite the fact that liquid (UV- and IR-)MALDI was introduced as early as solid MALDI [11, 13, 14] and liquid MALDI applications and methodologies have been continuously developed since then $[15,16]$.

This article shows that dramatic improvements can be made in the stable ion production for long and robust mass measurements, whereas the analytical sensitivity is within one order of magnitude of that achieved with solid-state matrices. The practical value of this type of performance can further extend the applicability of MALDI in biological mass spectrometry. For instance, absolute quantitation that is comparable with that of electrospray ionization (ESI), using MALDI ion signal intensities, can be achieved using the protocol presented here.

\section{Experimental}

\section{Materials}

Matrix compounds and peptides were purchased from Sigma-Aldrich (Poole, Dorset, UK), whereas solvents were obtained from Rathburn (Walkerburn, Scotland, 
UK). All chemicals, solvents, and peptides were used without further purification.

MALDI target plates were standard Waters Micromass 96-well MALDI sample plates (Waters Corporation, Manchester, UK). The target plates' front surface was covered with silicon adhesive conductive hydrophobic Teflon ${ }^{\mathrm{TM}}$ (polytetrafluoroethylene) tape (Product 20-3S, CS Hyde Company, Lake Villa, IL).

\section{MALDI Sample Preparation}

Small pieces of the adhesive conductive hydrophobic tape were applied to the sample plates to cover most of their front surfaces before MALDI samples were spotted on them.

For solid-state MALDI MS using DHB and CHCA as matrices an aliquot of $0.5 \mu \mathrm{L}$ analyte solution was mixed with $0.5 \mu \mathrm{L}$ of matrix solution on the hydrophobic tape and dried under ambient conditions. The DHB matrix solution was a saturated aqueous solution, whereas the CHCA matrix solution was prepared at a concentration of $0.5 \mathrm{~g} / \mathrm{L}$ in 2:1 (v:v) ethanol:acetonitrile.

For liquid MALDI MS, CHCA, 3-aminoquinoline, $10 \mathrm{mM}$ ammonium phosphate in 1:1 (v:v) water:methanol, and glycerol were mixed in a microcentrifuge tube in an approximately 1:3:5:5 ratio by weight. The mixture was slightly vortexed and sonicated until all compounds were dissolved and a clear yellow solution was obtained. Subsequently, the liquid matrix mixture was diluted 30-fold by adding equal volumes of methanol and aqueous 10-100 mM ammonium phosphate solution. The diluted liquid matrix was mixed with an equal volume of analyte solution and aliquots of 0.5 to $5 \mu \mathrm{L}$ were spotted on the hydrophobic Teflon ${ }^{\mathrm{TM}}$ tape and dried under ambient conditions.

\section{Instrumentation}

All measurements were performed on a Waters Micromass Q-TOF Premier mass spectrometer equipped with a standard MALDI source (Waters Corporation). The laser focus was adjusted to a spot size of about $200 \times$ $300 \mu \mathrm{m}$ as measured by ablation of a thin film of CHCA on a unmodified standard sample plate surface. Using burn marks in the hydrophobic tape on the MALDI sample plates a laser focus of about $50 \times 80 \mu \mathrm{m}$ was determined under otherwise identical conditions. Cooling gas was set to its maximum value. All spectra were acquired in the " $\mathrm{V}$ " mode with laser repetition rates between 5 and $20 \mathrm{~Hz}$.

\section{Quantitative Measurements}

For quantitation based on ion signal, mass spectra were acquired every $2 \mathrm{~s}$ for $3 \mathrm{~min}$ with a laser repetition rate of $5 \mathrm{~Hz}$. The first minute of acquisition was not included in the measurement. The spectra were smoothed by five passes through a Savitzky-Golay filter with a window of five channels and the peak intensities were used as a measure of the amount of sample in the droplet.

\section{Results and Discussion}

One of the advantages of the liquid MALDI matrix system and sample preparation technique as presented here is the possibility of concentrating the analyte by

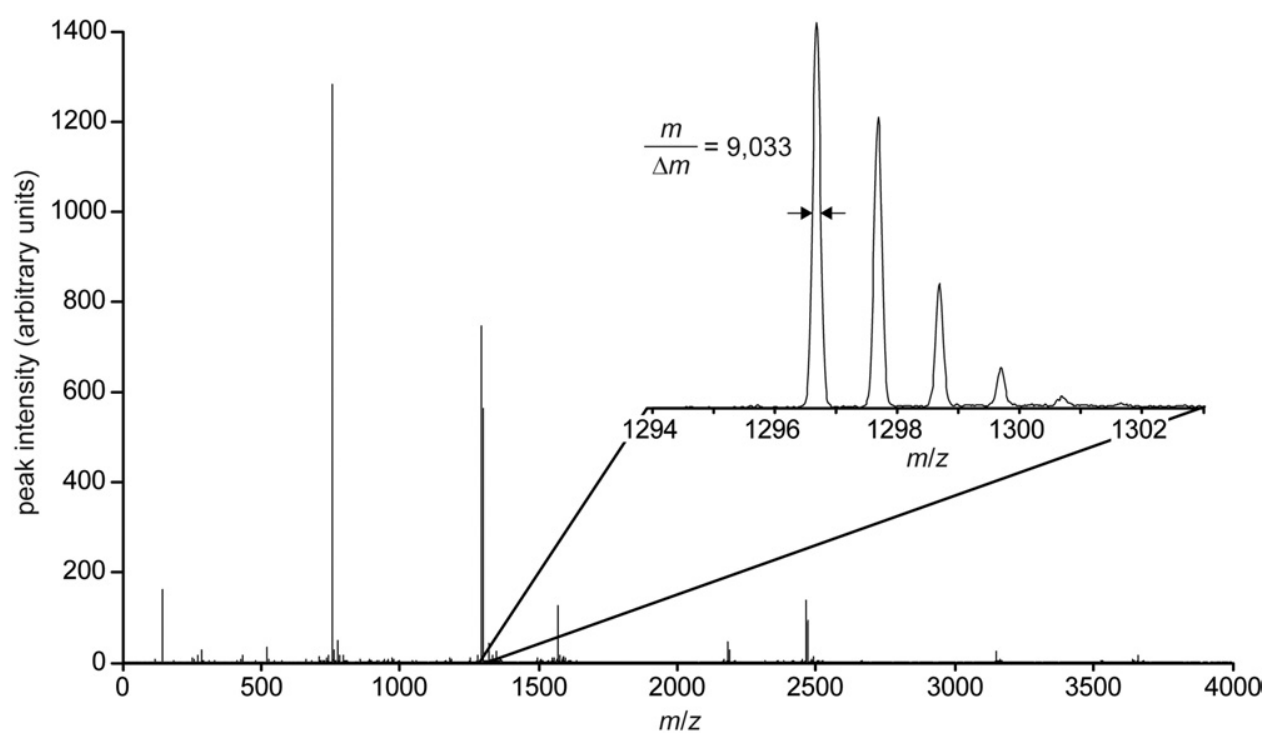

Figure 1. Liquid UV-MALDI mass spectrum of the peptide mixture used for quantitative and stability measurements, containing the tetrapeptide RFDS (524.2469 Da), bradykinin fragment 1-7 (757.3992 Da), angiotensin I (1296.6848 Da), substance P (1347.7354 Da), Glu-fibrinopeptide (1570.6773 Da), ANP 104-123 (2183.5500 Da), ACTH 18-39 (2465.1983 Da), Somatostatin (3147.4700 Da), and ACTH 7-38 (3657.9288 Da) at molar ratios 5:5:3:0.2:1:1:2:2:3. The resolving power is similar to that measured for solid matrices in the same instrument. 

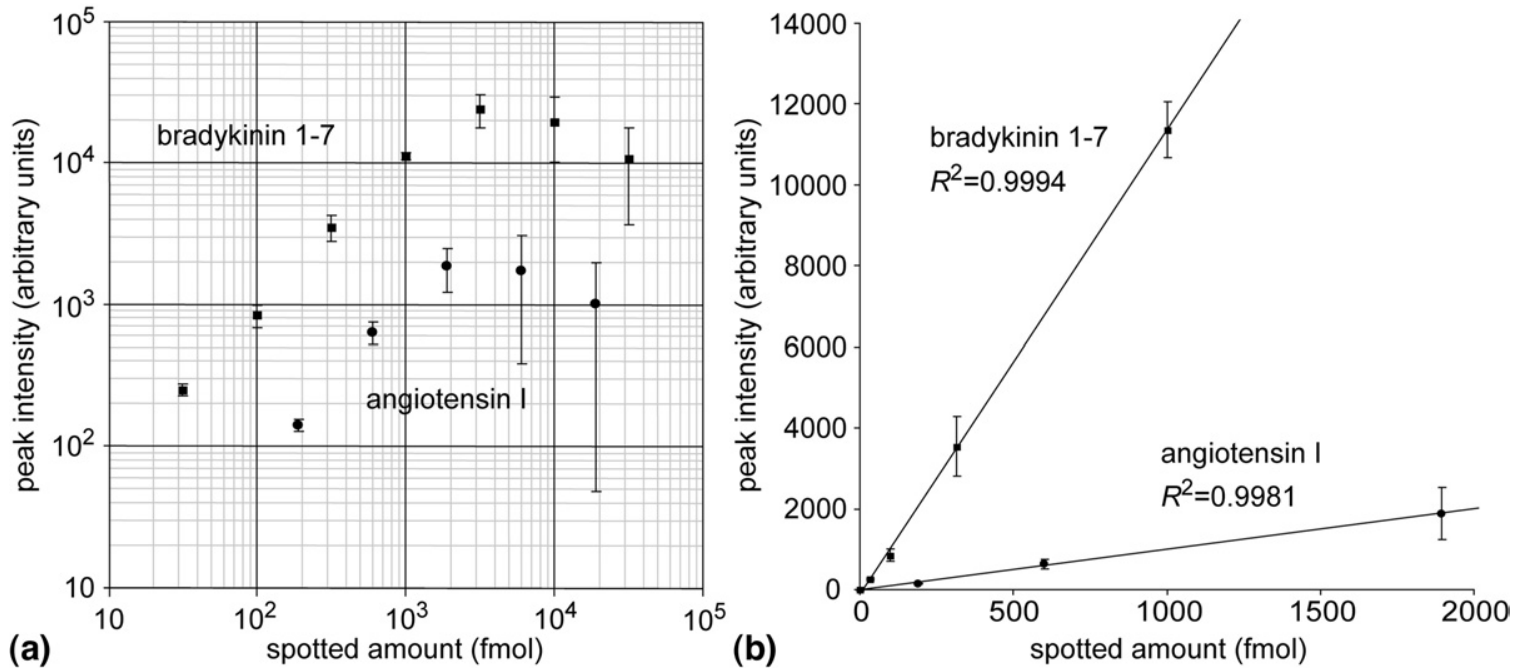

Figure 2. Dynamic range for absolute quantitation of bradykinin 1-7 (upper) and angiotensin I (lower) based on ion signal intensity (low to high femtomole deposited). The amount consumed during measurement is roughly $5 \%$ of the spotted amount. In general, four measurements were averaged at each concentration. The ion signals reach a maximum in the low picomole range and then decrease with concomitant increase in variance (A). The linear dynamic range determined with four measurements at each concentration (up to $\sim 1$ pmol deposited) is at least two orders of magnitude with linear correlation coefficients $R^{2}>0.998$ for bradykinin 1-7 and angiotensin I.

evaporation of the volatile matrix solvents, leading to high analyte concentration, as is the case with solidstate matrices after sample crystallization. Compared to most of the reported ionic liquid matrices it is a truly liquid MALDI matrix, even at low volumes and high

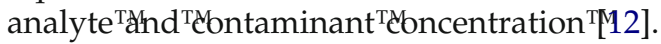

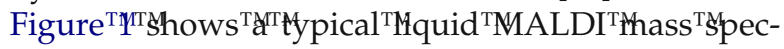
trum of the peptide mixture used for the measurements described here. In general, the protonated species dominated the ion signals, whereas only little alkali metal adduct ion formation was detected. The resolving power (>9000 at $m / z 1300)$ is within instrument specifications and similar to that measured for solid matrices. The ion signal in liquid MALDI MS is proportional to the concentration of analyte in the originally deposited droplet and can be used for absolute concentration over more than two orders of magnitude (low to high

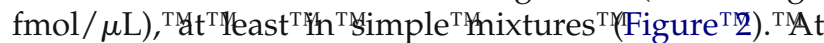
higher concentrations the signal is more variable and

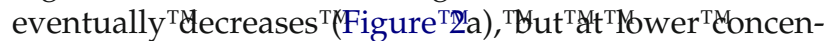

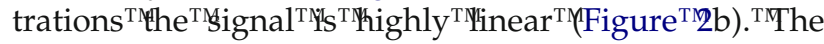
signal stability facilitates tuning of various parameters that previously could be achieved only by switching the

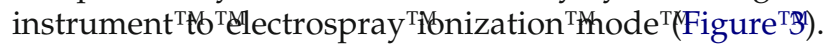
Changing ion sources on this instrument typically requires several hours of pump-down and detector conditioning. The signal from an originally deposited $2-\mu \mathrm{L}$ droplet lasted 30-70 min, whereas 5- $\mu \mathrm{L}$ droplets lasted

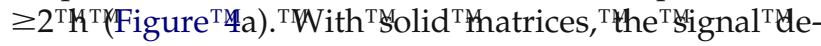
creases rapidly (over a few tens of laser shots) from a fixed desorption spot. Moving the target (that is, changing the desorption spot) can refresh the ion signal,
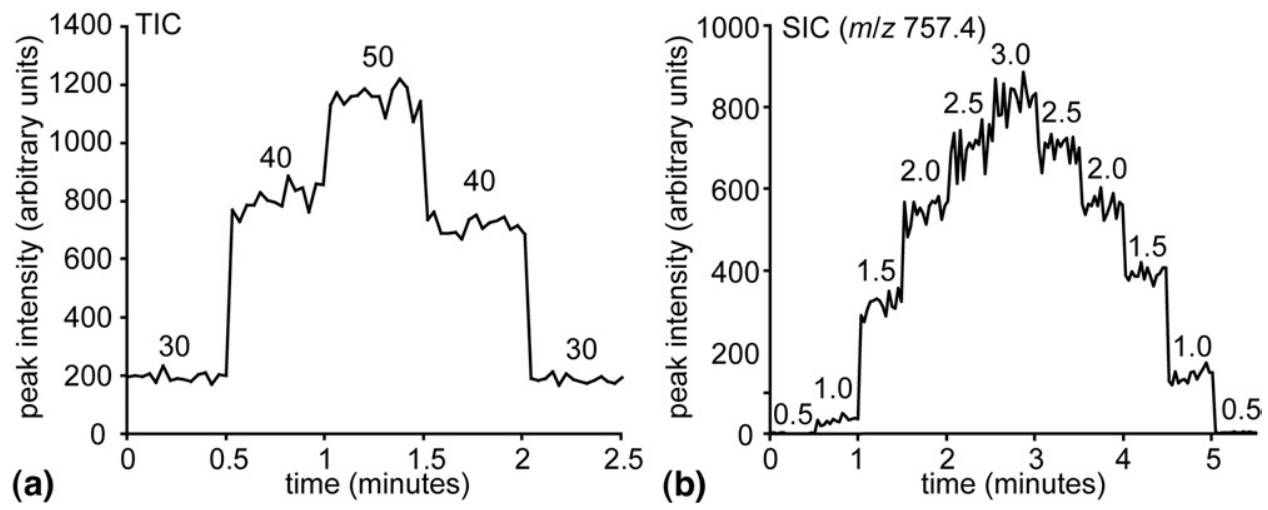

Figure 3. The stable ion current obtained with the liquid matrix can be used for tuning the Q-TOF instrument without time-consuming changing to electrospray ionization as illustrated by tuning the hexapole using total ion intensity (a) and the ion extraction using selected ion intensity (b). The parameters refer to the user-controlled voltage settings in the MassLynx ${ }^{\mathrm{TM}}$ software. 
although the high signal variation makes this a poor

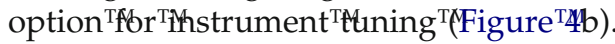

Quantitative liquid MALDI MS of peptides was previously demonstrated. So far the published data were collected mainly in the picomole range of analyte spotted on the target. The reported linear dynamic ranges of calibration curves were between one and two

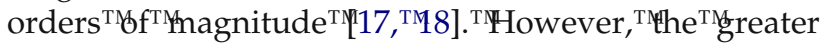
dynamic range of up to two orders of magnitude was achieved by relative measurement to an internal stan-

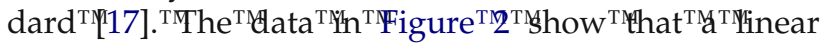
dynamic range of two orders of magnitude with a high $R^{2}$ value of $>0.998$ was obtained at the femtomole range without using an internal reference and, in contrast to

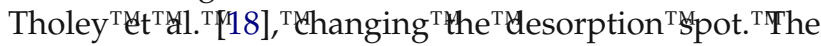
decrease of analyte ion signal intensity at increased analyte amount was previously observed with liquid and ${ }^{\mathrm{T}}$ Sblid ${ }^{\mathrm{T} M A L D I}$ TM8, T199].

The use of a disposable, inexpensive $(\sim 10 \notin$ per target plate) and commercially available adhesive Teflon ${ }^{\mathrm{TM}}$ tape eliminated carryover between samples and afforded an easy change of target surfaces. No sample contamination from the surface was observed, although fluoropolymer ions were desorbed from the bare surface in the absence of sample, particularly under high laser irradiance. The resistance of the Teflon ${ }^{\mathrm{TM}}$ tape was measured to be around $4 \mathrm{k} \Omega / \mathrm{cm}$ along the surface. Nonconductive films were also investigated, but with poor results.

After liquid MALDI droplets of nominally $0.5 \mu \mathrm{L}$ were dried down in air ( $\sim 30 \mathrm{~min})$, the droplet volume was determined to be about $16 \pm 5 \mathrm{~nL}$ measuring the droplets' diameters and assuming that the droplets resembled perfect half-spheres. However, after $24 \mathrm{~h}$ in the source vacuum without any laser desorption/ ablation, the droplets reduced in volume to $9 \pm 3 \mathrm{~nL}$. This is twice the volume based on the total volume of glycerol in the originally deposited sample droplets. For pictures and time-lapse videos of drying droplets, see the Supplementary Material section which can be found in the electronic version of this article.

Using sample droplets spotted at a volume of $2.0 \mu \mathrm{L}$ and analyzed at $5-\mathrm{Hz}$ laser repetition rate, the analyte ion signal was continuously seen for $>30$ min using $<200$ fmol total analyte amount. Thus, analyte consumption can be calculated to be in the low-attomole range per laser shot or low-femtomole range per minute, comparing well with analyte consumption in electrospray ionization. Based on the same data overall sample consumption is conservatively calculated to be $<1 \mathrm{pL}$ per laser shot.

\section{Conclusion}

The new liquid MALDI sample preparation method presented here shows great promise for quantitative MALDI MS measurements, similar to label-free quantitative methods based on electrospray ionization. The liquid matrix allows convenient tuning and optimization of the mass spectrometer and may enable many analyses that are not feasible with current solid MALDI matrices. The use of a conductive hydrophobic tape helps concentrate deposited microliter samples to droplets of a few nanoliters. The amount of glycerol in the

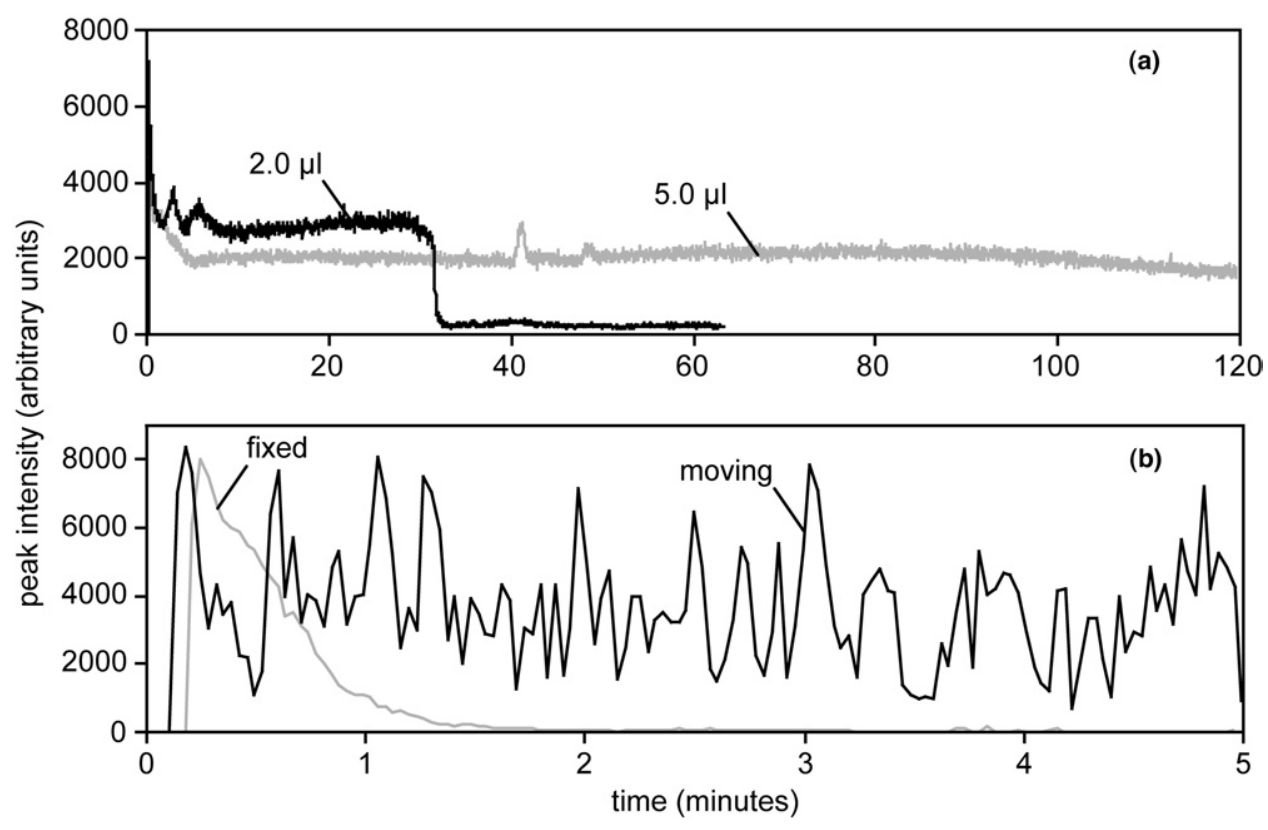

Figure 4. Stable ion signals (a) extend for 30-70 min from a 2- $\mu \mathrm{L}$ deposit (black). Larger deposits last longer, $1-2 \mathrm{~h}$ or more for a $5-\mu \mathrm{L}$ deposit (gray). The ion signals obtained with DHB as solid matrix (b) from a fixed position (gray) decay rapidly. Moving the target, that is, changing the desorption spot, extends the ion signal (black) but does not yield a stable ion signal profile and is unsuitable for instrument tuning. 
matrix preparation could be adjusted to achieve an appropriate degree of concentration depending on the sample. The cost of the hydrophobic tapes is negligible and using a disposable target surface eliminates carryover between analyses. Future development will include optimization of the liquid matrix composition for specific analyses or experiments.

\section{References}

1. Aebersold, R.; Mann, M. Mass Spectrometry-based Proteomics. Nature 2003, 422, 198-207.

2. Cramer, R.; Gobom, J.; Nordhoff, E. High-throughput Proteomics Using Matrix-assisted Laser Desorption/Ionization Mass Spectrometry. Expert. Rev. Proteomics 2005, 2, 407-420.

3. Jensen, O. N.; Mortensen, P.; Vorm, O.; Mann, M. Automation of Matrix-assisted Laser Desorption/Ionization Mass Spectrometry Using Fuzzy Logic Feedback Control. Anal. Chem. 1997, 69, 1706-1714.

4. Gobom, J.; Schuerenberg, M.; Mueller, M.; Theiss, D.; Lehrach, H.; Nordhoff, E. Alpha-cyano-4-hydroxycinnamic Acid Affinity Sample Preparation. A Protocol for MALDI-MS Peptide Analysis in Proteomics. Anal. Chem. 2001, 73, 434-438

5. Schuerenberg, M.; Luebbert, C.; Eickhoff, H.; Kalkum, M.; Lehrach, H.; Nordhoff, E. Prestructured MALDI-MS Sample Supports. Anal. Chem. 2000, 72, 3436-3442.

6. Gusev, A. I.; Wilkinson, W. R.; Proctor, A.; Hercules, D. M. Improvement of Signal Reproducibility and Matrix/Comatrix Effects in MALDI Analysis. Anal. Chem. 1995, 67, 1034-1041.

7. Sze, E. T.; Chan, T. W.; Wang, G. Formulation of Matrix Solutions for Use in Matrix-assisted Laser Desorption/Ionization of Biomolecules. I. Am. Soc. Mass Spectrom. 1998, 9, 166-174.

8. McCombie, G.; Knochenmuss, R. Enhanced MALDI Ionization Efficiency at the Metal-Matrix Interface: Practical and Mechanistic Consequences of Sample Thickness and Preparation Method. J. Am. Soc. Mass Spectrom. 2006, 17, 737-745.
9. Armstrong, D. W.; Zhang, L. K.; He, L.; Gross, M. L. Ionic Liquids as Matrixes for Matrix-assisted Laser Desorption/Ionization Mass Spectrometry. Anal. Chem. 2001, 73, 3679-3686.

10. Tholey, A.; Heinzle, E. Ionic (Liquid) Matrices for Matrix-assisted Laser Desorption/Ionization Mass Spectrometry-Applications and Perspectives. Anal. Bioanal. Chem. 2006, 386, 24-37.

11. Tanaka, K.; Waki, H.; Ido, Y.; Akito, S.; Yoshida, Y.; Yoshida, T. Protein and Polymer Analyses up to $m / z 100,000$ by Laser Ionization Time-offlight Mass Spectrometry. Rapid Commun. Mass Spectrom. 1988, 2, 151-153.

12. Cramer, R.; Corless, S. Liquid Ultraviolet Matrix-assisted Laser Desorption/Ionization-Mass Spectrometry for Automated Proteomic Analysis. Proteomics 2005, 5, 360-370.

13. Zhao, S.; Somayajula, K. V.; Sharkey, A. G.; Hercules, D. M. Hillenkamp, F.; Karas, M.; Ingendoh, A. Novel Method for Matrixassisted Laser Mass Spectrometry of Proteins. Anal. Chem. 1991, 63, $450-453$.

14. Overberg, A.; Karas, M.; Bahr, U.; Kaufmann, R.; Hillenkamp, F. Matrix-assisted Infrared-laser $(2.94 \mu \mathrm{m})$ Desorption/Ionization Mass Spectrometry of Large Biomolecules. Rapid Commun. Mass Spectrom. 1990, 4, 293-296.

15. Li, L.; Wang, A. P. L.; Coulson, L. D. Continuous-Flow Matrix-Assisted Laser Desorption Ionization Mass Spectrometry. Anal. Chem. 1993, 65, 493-495.

16. Cramer, R.; Burlingame, A. L. Employing Target Modifications for the Investigation of Liquid Infrared Matrix-assisted Laser Desorption/ Ionization Mass Spectrometry. Rapid Commun. Mass Spectrom. 2000, 14, 53-60.

17. Li, Y. L.; Gross, M. L. Ionic-Liquid Matrices for Quantitative Analysis by MALDI-TOF Mass Spectrometry. J. Am. Soc. Mass Spectrom. 2004, 15 1833-1837.

18. Tholey, A.; Zabet-Moghaddam, M.; Heinzle, E. Quantification of Peptides for the Monitoring of Protease-catalyzed Reactions by Matrixassisted Laser Desorption/Ionization Mass Spectrometry Using Ionic Liquid Matrixes. Anal. Chem. 2006, 78, 291-297.

19. Wang, B. H.; Dreisewerd, K.; Bahr, U.; Karas, M.; Hillenkamp, F. Gas-phase Cationization and Protonation of Neutrals Generated by Matrix-assisted Laser Desorption. J. Am. Soc. Mass Spectrom. 1993, 4 , 393-398. 